\title{
Open Issues in Agri-food Robot Standardization-the Red Meat Sector
}

\author{
Kristóf Takács \\ Antal Bejczy Center of Intelligent Robotics, EKIK \& \\ Óbuda University \\ Budapest, Hungary \\ kristof.takacs@irob.uni-obuda.hu \\ Luis Eduardo Cordova-Lopez \\ Norwegian University of Life Science (NMBU) \\ Ås, Norway \\ luis.eduardo.cordova-lopez@nmbu.no
}

Doctoral School of Applied Informatics and Applied Mathematics

\author{
Alex Mason \\ Norwegian University of Life Science (NMBU) \\ Ås, Norway \\ alex.mason@nmbu.no
}

\author{
Tamás Haidegger \\ Antal Bejczy Center of Intelligent Robotics, EKIK \& \\ John von Neumann Faculty of Informatics \\ Óbuda University \\ Budapest, Hungary \\ tamas.haidegger@irob.uni-obuda.hu
}

\begin{abstract}
Ensuring the safety of equipment, operator and the environment during robotic operation is paramount. Robotic systems are appearing in more and more professional service applications, while mechanic and control system components are evolving fast themselves, the legislation and standards regarding these topics are lagging behind. In connection with the RoBUTCHER project - which is a pioneer research effort employing industrial robots for completely automated slaughtering it was revealed that there are no particular standards regulating directly robotics applied to the agri-food application domain. More specifically, the meat industry and the red meat sector within has only seen hygienic standards regarding machinery, not considering human-robot collaboration or safe autonomous robot operation in the abattoirs. The purpose of this paper is to provide a general overview of the relevant standards (and similar guiding documents) that could be used as pathfinders during the development of inherently safe robotic systems. Exploring the standard and legislation landscape should offer some instrumental help regarding the foreseen certification process of meat processing robots and robot cells in the near future.

Index Terms-robot, meat processing, standardization, food robotics
\end{abstract}

\section{INTRODUCTION \& STRUCTURE}

In the European Union (EU), the CE mark (Conformité Européenne) must be obtained for every electrical product, certifying that the product complies with the essential

This work has received funding from the European Union's Horizon 2020 research and innovation programme under grant agreement No 871631, RoBUTCHER (A Robust, Flexible and Scalable Cognitive Robotics Platform).

It is partially supported by Project no. 2019-1.3.1-KK-2019-00007, provided by the National Research, Development and Innovation Fund of Hungary.

Kristof Takacs acknowledges the financial support of Óbuda University Doctoral School of Applied Informatics and Applied Mathematics.

T. Haidegger is supported through the New National Excellence Program of the Ministry of Human Capacities.

T. Haidegger is a Bolyai Fellow of the Hungarian Academy of Sciences, his research is partially supported by the EFOP-3.6.2-16-2017-00016 project. requirements of the relevant EU health, safety and environmental protection legislation. The approval procedure can be managed by the manufacturer (under the legal responsibility of the CEO), or by an independent certification body (called Notified Body, when registered in the EU). When a Notified Body assesses a system, their responsibility is to ensure the conformity of the product with the legal requirements (regulations) before being placed on the market.

Standards are all voluntary by default, based on an industry and academic expert consensus, codifying already existing good practices, methods and general requirements. Nevertheless, since they often mean the best available structured set of requirements toward e.g., the safety of a type of system, standards are often made the basis of regulations by lawmakers (e.g., the ISO/IEC 60601-1 became the basis of the EC Medical Device Directive). When Notified Bodies are dealing with a new system, they usually consider the noncompulsory standards' recommendations as well during their system assessment, therefore manufacturers and developers should consider those from the early periods of development on, since certifications increase competitiveness [1]. Increased autonomy of robotic systems has greatly ameliorated certification challenges, and only recently emerged standards have been able to address the safety concerns of those-in an application domain specific manner [2].

Standardization efforts have been extensive in the robotics domain for the past three decades [3]. ISO (International Organization for Standardization) standards have been traditionally providing guidance for safety in this domain and formed the basis of the European Commission Machinery Directive (ECMD) [4]. It has been a long professional debate to unambiguously define a robot and its components. The traditional ISO 8373 - Robots and robotic devices - Vocabulary 
standard under ISO first appeared in 1996, only referring to "Manipulating industrial robots", later extended to all robots (in the ISO sense) [5]. The responsible Technical Committee (TC) 299 has revised its official definition numerous times in the past years to incorporate all new domains and forms of robots. Their key distinguishing factors are autonomy, mobility and task oriented behaviour. The current ISO definition of a robot being:

"programmed actuated mechanism with a degree of autonomy to perform locomotion, manipulation or positioning”,

wherein autonomy is defined as:

"ability to perform intended tasks based on current state and sensing, without human intervention" [6].

Another holistic definition to a "robot" is given in the Encyclopedia of Robotics:

"A robot is a complex mechatronic system enabled with electronics, sensors, actuators and software, executing tasks with a certain degree of autonomy. It may be pre-programmed, teleoperated or carrying out computations to make decisions." [7]

Robotics is rapidly advancing in almost all possible application domains, now entering the agri-food industry as well [8]. A prime example, the robot system of the mentioned RoBUTCHER project (www.robutcher.eu) will carry out cutting and manipulation tasks supported by RGB-D cameras, AI, Virtual Reality and telemanipulation [9], [10]. Accordingly, the system will be unprecedented in complexity from the safety and legislation aspects. The robots will handle raw meat products intended for human consumption, therefore the risk of contamination being high due to the presence of the guts and intestines, and the applied end of arm tooling (EOAT) are designed for meat and bone cutting and gripping, making them highly dangerous for humans. Given the current approach for classification, the robot cell would be considered as a professional service robot application, still falling under the TC Machinery Directive (2006/42/EC) when considering ensuring safety of the system (Fig. 1).

This review mainly covers the ISO standards, since they are globally accepted, have been pioneers in the robotics field and commonly used in the industry. Moreover, ISO certification is often required by industrial customers due to its direct linkage to the EC MD. It is worth to mention however that ISO only develops the international standards and does not issue certificates, that is performed by external certification bodies. The two main possibilities - according to www.iso.org - are as follows:

- Certification - the provision by an independent body of written assurance (a certificate) that the product, service or system in question meets specific requirements.

- Accreditation - the formal recognition by an independent body, generally known as an accreditation body, that a certification body operates according to international standards.

Beside ISO standards, some related EU directives, guidelines and recommendations were also reviewed.

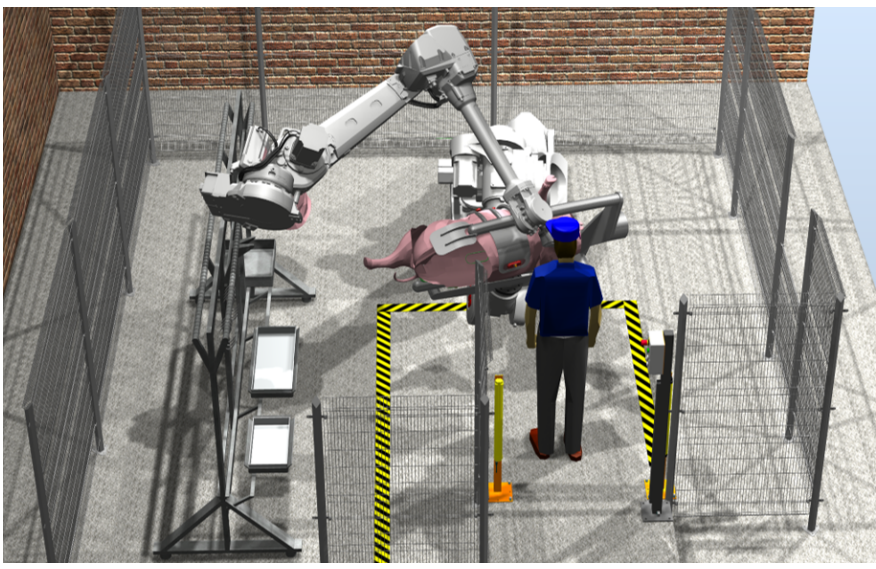

Fig. 1. Conceptual setup of the RoBUTCHER cell, able to autonomously or semi-autonomously (in a collaborative control mode) process entire pig carcasses. Image credit: RoBUTCHER project.

The clear and unambiguous use of the frequently occurring words and expressions in the robot industry is essential. ISO 8373 states that "This International Standard specifies vocabulary used in relation with robots and robotic devices operating in both industrial and non-industrial environments", thus this document will use the words and expressions according to this ISO standard's definitions [6]. ISO 8373 is currently under revision - a new version is in FDIS (Final Draft International Standard) status - thus some definitions may slightly change in the future.

\section{INDUSTRIAL ROBOTICS APPLIED IN THE MEAT SECTOR}

The RoBUTCHER project aims to develop the first entirely automated pig processing cell. This cell will carry out all steps of the pig-slaughtering with industrial robot arms (according to the EC MD), a motorized carcass handling unit, intelligent cutting and gripping tools, and some supplementary equipment, but most importantly, completely without any interaction from human operators. This huge change of approach in slaughterhouse-automation brings several different challenges not only from engineering aspects, but from safety and legislative aspects too. Robot industry is an especially fast evolving, ever-changing field, but since working together at any level with robots and/or machines is always potentially dangerous, several directives and strict standards apply to robotized solutions.

\section{A. Relevant documents}

Since there is no precedent in cell-based automated rawmeat handling or animal slaughtering using industrial robot arms, there is no single standard that regulates every aspects of this problem. To have a complete view on the standardization of the domain, the complete list of Robotistry was inspected for relevant standards, along with traditional online search engines [11]. Automated slaughtering seems to be such a special part of robotics, that even the Robotics 2020 Multi Annual Roadmap (MAR) does not cover this field in details [12]. The closest it gets to slaughterhouse automation is the 


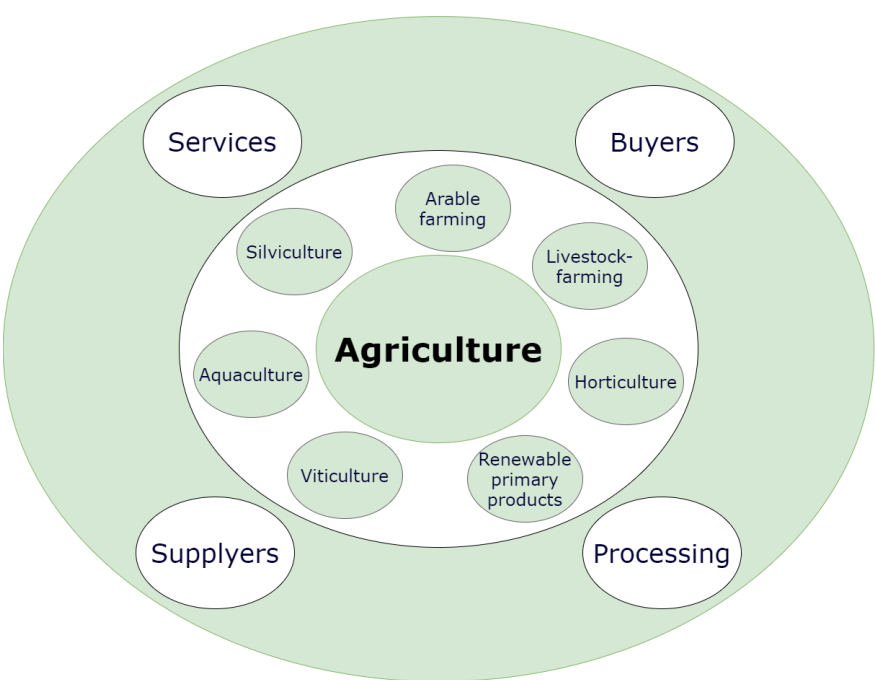

Fig. 2. Simplified structure of agricultural production categories. Although the Robotics 2020 MAR mentions the meat-sector in the agriculture section, it is not even presented as a subcategory. [12].

"Agriculture Domain" (chapter 2.4), defined as: "Agriculture is a general term for production of plants and animals by use of paramount natural resources (air, water, soil, minerals, organics, energy, information)."

However, slaughtering itself does not appear at all in the subcategories (Fig. 2), and even animals are barely mentioned within the document. The only appearance is in the "Food" section (2.2.10.2) under the "Manufacturing" sub-domains, where automation of deboning and raw-meat handling is mentioned. This makes solution developers' task particularly hard in the industry.

In spite of not addressing the meat-sector in depth, the statements and recommendations in the "Safety design and certification" section are worth considering. Most of them can be interpreted to the domain in question too, as well as some farming-related suggestions in the "Hardware in Loop" and the "Semantic Environment Awareness" sections, where simulations, planning systems, virtual models and semantic environment-representations are discussed.

The MAR document only provides guidelines and suggestions, while the acquisition of certifications is crucial in the food industry. Therefore, in this section the robot industry related standards will be discussed from here.

ISO/IEC started to work a decade ago on the integration of the new robotic application domains, and within the ISO/TC 299 Robotics technical committee, numerous working groups are active. One of the most important standards for almost all modern industrial solutions is the ISO 12100 Safety of machinery - General principles for design - Risk assessment and risk reduction, the latest version is the ISO 12100:2010 [13]. The primary purpose of this International Standard is to provide designers with an overall framework and guidance for decisions during the development of machinery to enable them to design machines that are safe for their intended use. However, the standard highlights in the "Scope" section that
"It does not deal with risk and/or damage to domestic animals, property or the environment."

ISO 12100:2010 also offers a classification of the related safety standards that will be used in this paper too [13]:

- Type-A standards (basic safety standards) giving basic concepts, principles for design and general aspects that can be applied to machinery;

- Type-B standards (generic safety standards) dealing with one safety aspect or one type of safeguard that can be used across a wide range of machinery:

- Type-B1 standards on particular safety aspects (for example, safety distances, surface temperature, noise);

- Type-B2 standards on safeguards (for example, two-hand controls, interlocking devices, pressuresensitive devices, guards);

- Type-C standards (machine safety standards) dealing with detailed safety requirements for a particular machine or group of machines.

In this sense, ISO 12100 is not specifically a standard for robotics, rather a more general standard (a type-A standard) covering a wide range of machinery design - including robotic applications. It is also intended to be used as a basis for the preparation of type-B or type-C safety standards, more specific to a given application.

The most relevant type-B (specifically type-B1) standard is the ISO11161:2007 Safety of machinery - Integrated manufacturing systems - Basic requirements [14]. As stated in the introduction, an integrated manufacturing system (IMS) can be very different in terms of size and complexity and can incorporate different technologies that require diverse expertise and knowledge, thus usually more specific (type-C) standards should be identified for the applications. The aim of this International Standard is to describe how to apply the requirements of ISO 12100-1:2003, ISO 12100-2:2003 (and ISO 14121 Safety of machinery, which is currently inactive, due to its integration into ISO 12100) in our specific context.

A more specific (type-C) robot industry related standard that could be applied to meat-industry automation is the ISO10218:2011 Robots and robotic devices - Safety requirements for industrial robots. This standard consists of 2 parts, Part 1: Robots and Part 2: Robot systems and integration [15], [16]. Part 2 is more suitable in this case, since meat handling requires EOAT and other external devices (e.g. sensors) forming a robot system together with the usually more than one robots, thus the robot-system specific problems apply too [17]. Nevertheless it relies on information presented in Part 1, thus Part 1 should also be taken into consideration. The relationship between the aforementioned ISO standards are shown in Fig. 3.

ISO/TR 20218-1:2018 Robotics - Safety design for industrial robot systems - Part 1: End-effectors can be also relevant, since potentially dangerous end-effectors are needed to carry out the cuttings (Part 2 is about manual load/unload stations, which is irrelevant in this case) [18]. The standard covers 


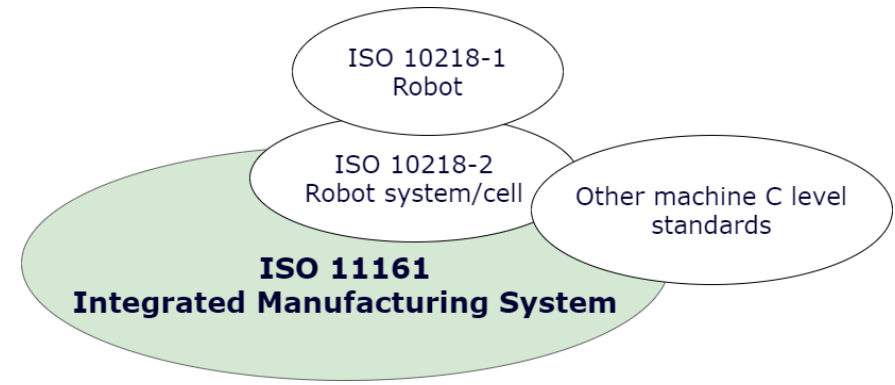

Fig. 3. Graphical view of relationships between standards relating to robot system/cell, emphasising the importance of the high-level (type A and B) standards. [16].

the collaborative cases too, where human operators and robot systems share the same workspace [19].

Regarding collaborative robotics, ISO/TR 15066:2015 Robots and robotic devices - Collaborative robots or ISO/TR 9241-810:2020 Ergonomics of human-system interaction shall be considered useful [20], [21]. However, the automated tools and devices (high payload industrial robots, saws, knives, not collaborative single purpose machines etc.) that usually take part in pig-slaughtering (i.e., cutting and tearing of human-like tissues and body parts) present an unacceptably high risk for any human in the workspace, no matter how strict the safety regulations are. Therefore, the RoBUTCHER project and this paper focuses on the completely automated slaughtering solutions, that excludes collaborativeness.

B. ISO 10218: Robots and robotic devices - Safety requirements for industrial robots

ISO 10218 is probably the most relevant standard for slaughterhouse automation, it specifies requirements and guidelines for inherent safe design, protective measures and information, basic hazards and requirements to reduce or eliminate the risks associated with them. Part 1 focuses on individual industrial robots, while Part 2 discusses robotsystems and their integration into a manufacturing system. A crucial statement is that the robot shall be designed in accordance with the principles of ISO 12100 for relevant hazards. It should also be mentioned, that although many sources of danger and possible hazardous scenarios are listed in the standard, it highlights that additional task-specific hazards might occur at every different application that also have to be examined.

Annex A of ISO 10218 presents a list of significant hazards, grouped by the type of hazards (mechanical, electrical etc.) along with examples, potential consequences and the relevant clause in the standard. According to the standard, a risk assessment shall be carried out on those hazards identified in the hazard identification process. This risk assessment shall give particular consideration to:

- The intended operations of the robot, including teaching, maintenance, setting and cleaning;

- Unexpected start-up;

- Access by personnel from all directions;

- Reasonably foreseeable misuse of the robot;
- The effect of failure in the control system;

- Where necessary, the hazards associated with the specific robot application.

Risks shall be eliminated or reduced first by design or by substitution, then by safeguarding and other complementary measures. Any residual risks shall then be reduced by other measures (e.g., warnings, signs, training).

The standard also suggests solutions in many relevant topics, such as robot stopping functions, power loss, actuating controls, speed control, singularity protection, axis limiting and safety-related control system performance. There is also a dedicated chapter ("Information for use") that helps with the preparation of a proper documentation (an instruction handbook), with suggested marking, symbols, etc.

Detailed descriptions, lists and specific instructions can be found in the annexes of the standard, that are:

- Annex A: List of significant hazards

- Annex B: Stopping time and distance metric

- Annex C: Functional characteristics of three-position enabling device

- Annex D: Optional features

- Annex E: Labelling

- Annex F: Means of verification of the safety requirements and measures.

Part 2 of ISO 10218 states that "the design of the robot system and cell layout is a key process in the elimination of hazards and reduction of risks" [16]. In accordance with this, Part 2 suggests basic layout design principles about physical limits, workspaces, manual intervention, ergonomics, human interfacing, perimeter safeguarding, etc.

Parts of a robot system usually fall under the scope of other standards, thus ISO 10218 provides a useful list of those, also to be considered by new product developers:

- Equipotential bonding/earthing requirements (grounding): IEC 60204-1.

- Electric power: IEC 60204-1

- Hydraulic power: ISO 4413

- Pneumatic power: ISO 4414

- Actuating control: IEC 60204-1

- Emergency stop function: IEC 60204-1, ISO 13850, IEC 61800-5-2.

- Lighting: ISO 8995-1

- Enabling devices: ISO 10218-1-Annex D

C. ISO/DTR 20218-1:2018 Robotics - Safety design for industrial robot systems - Part 1: End-effectors

ISO 20218-1 is a Technical Report (TR) offering guidance to indicate safety measures for the design and the integration of end-effectors used for robot systems. This includes the manufacturing, design, and integration of end-effectors, and the necessary information for use. Part 2 of ISO 20218 is dealing with manual load and unload stations, that is out of scope considering slaughterhouse automation.

The standard suggests to avoid sharp edges and generally dangerous structures on EOAT, however knives or saws are 
necessary in meat-processing, thus the only option is riskminimization. For this purpose protective devices and builtin safety-related control systems are presented, such as force sensing, speed monitoring, presence sensing, emergency stop, etc. Besides sharp tools like knives, there is a separate section dedicated for gripper end-effectors mentioning grasp-type grippers (force closure and form closure too), vacuum grippers and magnet grippers. The first two are commonly used in the meat-industry too [22].

ISO 20218 also has annexes with references to other possibly relevant standards and practical risk assessment scenarios. Furthermore, there are examples for safety-rated monitored stopping, gripper designs and their safety performance and a table about hazards, their potential origins and consequences, similar to the one in ISO 10218.

\section{FOOD-SAFETY STANDARDS}

Beside the safety of robots and automated machinery, foodsafety is also important in automated slaughterhouses, however this paper focuses on the safety and legislation aspects of robotics. Food safety as an expression is defined as:

"Assurance that food will not cause an adverse health effect for the consumer when it is prepared and/or consumed in accordance with its intended use" [23].

The adoption of a Food Safety Management System (FSMS) is a strategic decision that can help to improve a project's overall performance in food safety [24]. The potential benefits to an organization or project of implementing an FSMS are:

- the ability to consistently provide safe foods and products and services that meet customer and applicable statutory and regulatory requirements;

- addressing risks associated with its objectives;

- the ability to demonstrate conformity to specified FSMS requirements.

The most general ISO standard in this topic is the ISO 22000:2018 Food safety management systems - requirements for any organization in the food chain [23]. The standard adopts a process approach - a detailed plan-do-check-act (PDCA) cycle - when developing and implementing a FSMS and improving its effectiveness to enhance production of safe products and services while meeting applicable requirements.

The technical parts of the standard discuss the role and main tasks of the organization, the PDCA cycle, the operation, communication and documentation required to maintain the safe operation. The standard also covers hazard control, analysis and assessment, emergency response, monitoring and measuring. The last parts offer recommendations about internal auditing, management review and continual improvement.

Some of the mentioned and likely relevant standards and other documents are the followings:

- ISO/TS 22002 Prerequisite programmes on food safety;

- ISO/TS 22003 Food safety management systems - Requirements for bodies providing audit and certification of food safety management systems;
- ISO 22005 Traceability in the feed and food chain General principles and basic requirements for system design and implementation;

- CAC/RCP 1-1969 General Principles of Food Hygiene;

- Joint FAO/WHO Food Standards Programme. Codex Alimentarius Commission: Procedural Manual.

It is notable to mention that the agROBOfood project was established in 2019 by the EU as a Digital Innovation Hub (DIH), partially to facilitate and streamline standardization activities in the near future [25]. The agROBOfood consortium of 39 partners aims to accelerate the sector's digital transformation through the adoption of robotic technologies.

\section{Discussion}

While there are numerous robot safety standards for the traditional industrial applications, there are very few for the service robot domains, and technically none for the automated meat processing industry - or similar. To facilitate the development of new prototypes and products, and the clearance of the automated meat-processing plants (such as the RoBUTCHER Meat Factory Cell), safety considerations shall follow the general minimum hazard principle (identifying and reducing the hazards), based on the existing standards (Fig. 1).

It is likely that following the safety design principles of ISO 10218 standards family, a systematic solution can be given to most system structures. Nevertheless, the chosen Notified Body for certification might have other and additional requirements. The agri-food domain may well adapt existing safety requirement structures from other application areas, such as medical robotics, where the Degree of Autonomy and Level of Autonomy for a system have been linked to different safety requirements [26] [2]. It is reasonable to assume that choosing the maximum safety control principle of the robot cell (e.g., teleoperation instead of collaborative control for exception management and manual override) significantly increases the future applicability/deployability of such developments.

\section{REFERENCES}

[1] A. Okanovic, B. T. Jokanovic, V. Dakovic, S. Vukadinovic, and J. S. Jesic, "Innovating a model for measuring competitiveness in accordance with the challenges of industry 4.0," Acta Polytechnica Hungarica, vol. 17, pp. 67-88, 2020.

[2] K. Chinzei, "Safety of surgical robots and IEC 80601-2-77: The first international standard for surgical robots," Acta Polytechnica Hungarica, vol. 16, pp. 171-184, 2019.

[3] T. Jacobs, J. Veneman, G. Virk, and T. Haidegger, "The flourishing landscape of robot standardization [industrial activities]," IEEE Robotics \& Automation Magazine, vol. 25, pp. 8-15, 032018.

[4] "Directive 2006/42/ec of the european parliament and of the council of 17 may 2006 on machinery, and amending directive 95/16/ec," European Parliament, Council of the European Union, Directive, 17/05/2006.

[5] "ISO 8373:1994/COR Manipulating industrial robots — vocabulary — technical corrigendum 1," International Organization for Standardization, ISO, 1996.

[6] "ISO 8373:2012 Robots and robotic devices — vocabulary," International Organization for Standardization, ISO, March 2012.

[7] T. Haidegger, "Taxonomy and standards in robotics," in Encyclopedia of Robotics, O. K. Marcelo H. Ang and B. Siciliano, Eds. Springer Nature, 2021, pp. 1-10 (in press). 
[8] G. Haidegger and I. Paniti, "Episodes of robotics and manufacturing automation achievements from the past decades and vision for the next decade," Acta Polytechnica Hungarica, vol. 16, no. 10, pp. 119-136, 2019.

[9] O. Alvseike, H. Sverdvik, M. O'Farrell, and P. Berg, "Meat factory cell-a concept for the future?" in 63rd International Congress of Meat Science and Technology: Nurturing locally, growing globally. Wageningen Academic Publishers, 2017.

[10] I. de Medeiros Esper, A. Mason et al., "Robotisation and intelligent systems in abattoirs," Trends in Food Science \& Technology, vol. 111, no. XX, pp. 1-15, 2021.

[11] robotistry.org, "Aggregated standards list," 2015. [Online]. Available: http://robotistry.org/standards/StandardsList.html

[12] "Robotics 2020 multi-annual roadmap," Horizon 2020 Call ICT-2016 (ICT-25 \& ICT-26). [Online]. Available: "https://www.eurobotics.net/cms/upload/press/2015/files/H2020_Robotics_MultiAnnual_Roadmap_ICT-2016.pdf"

[13] "ISO 12100:2010 Safety of machinery — general principles for design - risk assessment and risk reduction," International Organization for Standardization, ISO, November 2010.

[14] "ISO 11161:2007 Safety of machinery — integrated manufacturing systems - basic requirements," International Organization for Standardization, ISO, May 2007.

[15] "ISO 10218-1:2011 Robots and robotic devices — Safety requirements for industrial robots - Part 1," International Organization for Standardization, ISO, July 2011.

[16] "ISO 10218-2:2011 Robots and robotic devices — safety requirements for industrial robots - part 2: Robot systems and integration," International Organization for Standardization, ISO, July 2011.

[17] M. Turanli and H. Temeltas, "Multi-robot workspace allocation with hopfield networks and imprecise localization," Acta Polytechnica Hungarica, vol. 17, pp. 169-188, 012020.

[18] "ISO 20218-1:2018 Robotics - safety design for industrial robot systems - part 1: End-effectors," International Organization for Standardization, ISO/TR 20218-1:2018, August 2018.

[19] J. Bessler, L. Schaake, C. Bidard, J. Buurke, A. Lassen, K. Nielsen, J. Saenz, and F. Vicentini, COVR â€" Towards Simplified Evaluation and Validation of Collaborative Robotics Applications Across a Wide Range of Domains Based on Robot Safety Skills: Proceedings of the 4th International Symposium on Wearable Robotics, WeRob2018, October 16-20, 2018, Pisa, Italy, 01 2019, pp. 123-126.

[20] "ISO 15066:2016 Robots and robotic devices - collaborative robots," International Organization for Standardization, ISO, February 2016

[21] "ISO 9241-810:2020 Ergonomics of human-system interaction - part 810: Robotic, intelligent and autonomous systems," International Organization for Standardization, ISO/TR, September 2020.

[22] K. Takács, A. Mason, L. B. Christensen, and T. Haidegger, "Robotic grippers for large and soft object manipulation," in 2020 IEEE 20th International Symposium on Computational Intelligence and Informatics (CINTI), 2020, pp. 133-138.

[23] "ISO 22000:2018 Food safety management systems — requirements for any organization in the food chain," International Organization for Standardization, ISO, June 2018.

[24] A. Panghal, N. Chhikara, N. Sindhu, and S. Jaglan, "Role of food safety management systems in safe food production: A review," Journal of food safety, vol. 38, no. 4, p. e12464, 2018.

[25] C. Lokhorst, E. Pekkeriet, and G. Chatzikostas, "agrobofood: A paneuropean digital innovation hub network for robotics in agri-food systems," Feb. 2020.

[26] T. Haidegger, "Autonomy for Surgical Robots: Concepts and Paradigms," IEEE Transactions on Medical Robotics and Bionics, vol. 1, no. 2, pp. 65-76, May 2019. 